\title{
Position of the maximum in a sequence with geometric distribution
}

\author{
Margaret Archibald ${ }^{1}$ \\ ${ }^{1}$ The John Knopfmacher Centre for Applicable Analysis and Number Theory, School of Mathematics, University of \\ the Witwatersrand, Private Bag 3, Wits 2050, Johannesburg, South Africa. marchibaldemaths. wits.ac. za
}

As a sequel to [1], the position of the maximum in a geometrically distributed sample is investigated. Samples of length $n$ are considered, where the maximum is required to be in the first $d$ positions. The probability that the maximum occurs in the first $d$ positions is sought for $d$ dependent on $n$ (as opposed to $d$ fixed in [1]). Two scenarios are discussed. The first is when $d=\alpha n$ for $0<\alpha \leq 1$, where Mellin transforms are used to obtain the asymptotic results. The second is when $1 \leq d=o(n)$.

Keywords: Mellin transforms, generating functions, geometric distribution.

\section{Introduction}

Consider a word whose letters are natural numbers. Assume that these letters occur independently and with geometric probability. So for $p+q=1$, each letter $j$ appears in the word with probability $p q^{j-1}$. We write $Q:=q^{-1}$ and $L:=\log Q$.

We address the question: "What is the probability that the maximum in a word occurs in the first $d$ positions?" We take words of length $n$ and require $d \leq n$. Previously (in [1]), $d$ was considered fixed. Now, $d$ is allowed to grow with $n$. First, we assume that $d$ is proportional to $n$ and then we consider the case when $d$ is $o(n)$ (but at least 1). The latter produces the same solutions as when $d$ is fixed (see [1]).

We distinguish between two cases: 'strict' and 'weak'. A 'strict' maximum never recurs, whereas a 'weak' maximum can recur any number of times. These apply separately to the two parts of the word (the first $d$ letters, and the remaining $n-d$ letters). This means that in total, there are four different cases to be dealt with: (strict, strict), (weak, strict), (strict, weak) and (weak, weak), where the first entry refers to the first $d$ letters in the word, and the second entry to the rest of the word. The results in the (strict, strict) case for $d$ fixed will hold for the other scenarios too (i.e., it is not required that $d$ is independent of $n$ ). This is because the relevant calculations in [1] still go though when we take the limits as $n \rightarrow \infty$. This is not true for the remaining three cases which are dealt with in Section 3.

\section{Results}

Theorem 1 The probability that the maximum value in a word of length $n$ is in the first $d$ positions (for $d=\alpha n)$ is

$$
\begin{gathered}
\operatorname{Max}_{(w, s)}(n) \sim \frac{1}{L} \log \left(\frac{1}{1-\alpha\left(1-Q^{-1}\right)}\right)+\frac{1}{L}\left(\psi_{0}(n(1-\alpha p))-\psi_{0}(n)\right), \\
\operatorname{Max}_{(s, w)}(n) \sim \frac{\alpha(Q-1)}{L(1+\alpha(Q-1))}(1+\psi(n(q+p \alpha))), \\
\operatorname{Max}_{(w, w)}(n) \sim \frac{\log (1+\alpha(Q-1))}{L}+\frac{1}{L}\left(\psi_{0}(n)-\psi_{0}\left(n\left(\frac{q+\alpha p}{q}\right)\right)\right),
\end{gathered}
$$

as $n \rightarrow \infty$ where $Q:=q^{-1}, L:=\log Q$, and the fluctuations are defined by

$$
\psi(x):=\sum_{k \neq 0} \Gamma\left(1-\chi_{k}\right) e^{2 k \pi \boldsymbol{i} x}, \quad \text { for } \quad \mathrm{k} \in \mathbb{Z},
$$


and

$$
\psi_{0}(x):=\sum_{k \neq 0} \Gamma\left(-\chi_{k}\right) e^{2 k \pi i \log _{Q} x}, \quad \text { for } \quad \mathrm{k} \in \mathbb{Z} .
$$

Note that we write $i$ rather than the $i$ we use as an index.

Theorem 2 The probability that the maximum value in a word of length $n$ is in the first $d$ positions, for $1 \leq d=o(n)$, is

$$
\begin{aligned}
\operatorname{Max}_{(w, s)}(n) & \sim \frac{\left(1-Q^{-1}\right) d}{L n}(1+\psi(n)), \\
\operatorname{Max}_{(s, w)}(n) & \sim \frac{(Q-1) d}{L n}(1+\psi(n)), \\
\operatorname{Max}_{(w, w)}(n) & \sim \frac{(Q-1) d}{L n}(1+\psi(n)),
\end{aligned}
$$

as $n \rightarrow \infty$, where $\psi(x)=\sum_{k \neq 0} \Gamma\left(1-\chi_{k}\right) e^{2 k \pi i \log _{Q} x}, k \in \mathbb{Z}$.

\section{Suppose $d=\alpha n$}

Suppose we now consider $d=\alpha n$ where $0<\alpha \leq 1$. The ' $d$ fixed' results from [1] continue to hold only in the (strict, strict) case. For the other three cases we make use of a technique from complex analysis called the 'Mellin' transform. The rules used below can be found in [2] and [5], among others. The first case is done in greater detail than the other two, as a similar process is used in each case.

\subsection{Case (weak, strict), for $\boldsymbol{d}=\boldsymbol{\alpha} n$}

For this scenario (finding the probability with which the maximum, $k$, occurs in the first $d$ letters of a word) we require that there is at least one $k$ in the first $d$ places, and possibly more. However, the rest of the word may only have letters from the set $\{1, \cdots, k-1\}$. Now suppose that $d=\alpha n$, for $0<\alpha \leq 1$. The generating function is explained in [1], yielding the following coefficients (with $d$ replaced by $\alpha n$ ).

$$
f_{(w, s)}(n):=\sum_{k \geq 1} \sum_{i=0}^{\alpha n-1}\left(1-q^{k-1}\right)^{i+n-\alpha n} p q^{k-1}\left(1-q^{k}\right)^{\alpha n-1-i} .
$$

Now note that

$$
\sum_{i=0}^{\alpha n-1}\left(\frac{1-q^{k-1}}{1-q^{k}}\right)^{i}=\frac{\left(1-q^{k}\right)^{\alpha n}-\left(1-q^{k-1}\right)^{\alpha n}}{\left(1-q^{k}\right)^{\alpha n-1} q^{k-1}(1-q)},
$$

and thus, since $p=1-q$ and $(1-a)^{n} \sim e^{-a n}$ for small $a$,

$$
\begin{aligned}
f_{(w, s)}(n) & =\sum_{k \geq 1}\left(1-q^{k-1}\right)^{n(1-\alpha)}\left[\left(1-q^{k}\right)^{\alpha n}-\left(1-q^{k-1}\right)^{\alpha n}\right] \\
& \sim \sum_{k \geq 1}\left[e^{-n q^{k-1}(1-\alpha p)}-e^{-n q^{k-1}}\right] .
\end{aligned}
$$

We are now in a position to use Mellin transforms to find an approximation. We shift the fundamental strip from $\langle 0, \infty\rangle$ to $\langle-1,0\rangle$ and define a new function:

$$
f_{w s}(x):=\sum_{k \geq 1}\left[\left(e^{-n q^{k-1}(1-\alpha p)}-1\right)-\left(e^{-n q^{k-1}}-1\right)\right] .
$$

Then the Mellin transform of this function is:

$$
\begin{aligned}
f_{w s}^{*}(s) & =\sum_{k \geq 1}\left[\left(q^{k-1}(1-\alpha p)\right)^{-s} \Gamma(s)-\left(q^{k-1}\right)^{-s} \Gamma(s)\right] \\
& =\sum_{k \geq 1} q^{s(1-k)} \Gamma(s)\left[(1-\alpha p)^{-s}-1\right] \\
& =q^{s} \Gamma(s)\left[(1-\alpha p)^{-s}-1\right] \sum_{k \geq 1}\left(q^{-s}\right)^{k} \\
& =\Gamma(s)\left[(1-\alpha p)^{-s}-1\right] \frac{1}{1-q^{-s}}, \quad \text { for } \Re(s)<0,
\end{aligned}
$$


where $\Re(s)$ represents the real part of the complex number $s$. The reason for shifting the fundamental strip is that the transform exists in the intersection of the domain of convergence of the generalised Dirichlet series and the fundamental strip of $f^{*}(s)$. The intersection $\langle-\infty, 0\rangle \cap\langle 0, \infty\rangle$ is empty, but with the shift we have a final fundamental strip of $\langle-1,0\rangle$. We choose a value inside this, say $-\frac{1}{2}$, with which to perform our inverse Mellin transform:

$$
f_{w s}(x)=\frac{1}{2 \pi i} \int_{\left(-\frac{1}{2}\right)} \Gamma(s)\left[(1-\alpha p)^{-s}-1\right] \frac{1}{1-q^{-s}} x^{-s} d s .
$$

The notation $\left(-\frac{1}{2}\right)$ under the integral sign means an integral from $-\frac{1}{2}-i \infty$ to $-\frac{1}{2}+i \infty$. This can be approximated by moving the contour to the right (and thus collecting negative residues) since we are interested in $x$ large. The first poles we encounter are the simple pole at $s=0$ (which would be a double pole except that one cancels with the factor $\left.(1-\alpha p)^{-s}-1\right)$ and the simple poles at $s=\chi_{k}:=\frac{2 k \pi i}{L}$, $k \in \mathbb{Z} \backslash\{0\}$ where $L:=\log Q$. The former contributes the main term and the rest contribute the fluctuations which are comparatively extremely small. As $s \rightarrow 0$,

$$
\begin{gathered}
\Gamma(s) \sim \frac{1}{s}, \\
(1-\alpha p)^{-s}-1 \sim 1-s \log (1-\alpha p)-1=-s \log (1-\alpha p), \\
\frac{1}{1-q^{-s}}=\frac{1}{1-e^{-s \log q}} \sim \frac{1}{1-(1-s \log q)}=\frac{1}{s \log q},
\end{gathered}
$$

and

$$
x^{-s}=e^{-s \log x} \sim 1
$$

Thus the negative residue is

$$
-\left[s^{-1}\right] \frac{1}{s}(-s \log (1-\alpha p)) \frac{1}{s \log q}=\frac{\log (1-\alpha p)}{\log q} .
$$

We also have simple poles at $s=\chi_{k}$, for $k \neq 0$. Let $\varepsilon:=s-\chi_{k}$ then expanding around $\varepsilon=0$ gives

$$
\frac{1}{1-q^{-s}}=\frac{1}{1-q^{-\chi_{k}-\varepsilon}}=\frac{1}{1-q^{-\varepsilon}}=\frac{1}{1-e^{-\varepsilon \log q}} \sim \frac{1}{1-(1-\varepsilon \log q)}=\frac{1}{\varepsilon \log q} .
$$

So the negative residues for all non-zero $k$ are

$$
\begin{aligned}
\sum_{k \neq 0}(-1)\left[\varepsilon^{-1}\right] \Gamma\left(\chi_{k}\right)\left[(1-\alpha p)^{-\chi_{k}}-1\right] \frac{1}{\varepsilon \log q} x^{-\chi_{k}} & =\frac{1}{L} \sum_{k \neq 0} \Gamma\left(\chi_{k}\right)\left[(1-\alpha p)^{-\chi_{k}}-1\right] x^{-\chi_{k}} \\
& =\frac{1}{L} \sum_{k \neq 0} \Gamma\left(\chi_{k}\right)\left[e^{-\chi_{k} \log (1-\alpha p)}-1\right] e^{-\chi_{k} \log x} \\
& =\frac{1}{L} \sum_{k \neq 0} \Gamma\left(\chi_{k}\right)\left[e^{-\chi_{k} \log (x(1-\alpha p))}-e^{-\chi_{k} \log x}\right] \\
& =\frac{1}{L} \sum_{k \neq 0} \Gamma\left(-\chi_{k}\right)\left[e^{2 k \pi i \log _{Q}(x(1-\alpha p))}-e^{2 k \pi i \log _{Q} x}\right] .
\end{aligned}
$$

Now put these together to get the probability of having a weak maximum in the first $d$ positions which does not repeat in the rest of the word and where $d=\alpha n$ grows with $n$ as $n \rightarrow \infty$ :

$$
\begin{aligned}
& \frac{\log (1-\alpha p)}{\log q}+\frac{1}{L} \sum_{k \neq 0} \Gamma\left(-\chi_{k}\right)\left[e^{2 k \pi i \log _{Q}(n(1-\alpha p))}-e^{2 k \pi i \log _{Q} n}\right] \\
& =\frac{\log \left(1-\alpha\left(1-Q^{-1}\right)\right)}{-L}+\frac{1}{L}\left(\psi_{0}(n(1-\alpha p))-\psi_{0}(n)\right)
\end{aligned}
$$

for $Q=q^{-1}, L=\log Q$ and $\psi_{0}(x)$ as in Theorem 1. Note that if $\alpha=1$ then $d=n$ and the main term yields a probability of 1 , confirming our result on a word with no restrictions. 


\subsection{Case (strict, weak), for $d=\alpha n$}

In this case, we allow the maximum, $k$, to occur only once in the first $d$ letters, but any number of times in the rest of the word. Again, [1] provides us with the generating function whose coefficients are

$$
\begin{aligned}
f_{(s, w)}(n) & =\sum_{k \geq 1} \alpha n p q^{k-1}\left(1-q^{k-1}\right)^{\alpha n-1}\left(1-q^{k}\right)^{n(1-\alpha)} \\
& \sim \sum_{k \geq 1} \alpha n p q^{k-1} e^{-n q^{k-1}(q+p \alpha)}
\end{aligned}
$$

If we define the function

$$
f_{s w}(x):=\sum_{k \geq 1} \alpha x p q^{k-1} e^{-x q^{k-1}(q+p \alpha)},
$$

then the Mellin transform will be

$$
\begin{aligned}
f_{s w}^{*}(s) & =\sum_{k \geq 1} \alpha p q^{k-1}\left(q^{k-1}\right)^{-(s+1)}(q+p \alpha)^{-(s+1)} \Gamma(s+1) \\
& =\alpha p(q+p \alpha)^{-(s+1)} \Gamma(s+1) \frac{1}{1-q^{-s}}, \quad \text { for } \Re(s)<0,
\end{aligned}
$$

and the fundamental strip is the overlap of the interval $(-\infty, 0)$ and the fundamental strip of $x e^{-x}$ which is $\langle-1, \infty\rangle$, i.e., $\langle-1,0\rangle$. Hence we pick our contour integral from $-\frac{1}{2}-\boldsymbol{i} \infty$ to $-\frac{1}{2}+\boldsymbol{i} \infty$, and perform the inverse Mellin transform to get:

$$
f_{s w}(x)=\frac{1}{2 \pi \boldsymbol{i}} \int_{\left(-\frac{1}{2}\right)} \alpha p(q+p \alpha)^{-(s+1)} \Gamma(s+1) \frac{1}{1-q^{-s}} x^{-s} d s .
$$

By moving the contour to the right, the first poles we reach are at $s=0$ and $s=\chi_{k}, k \neq 0$. For the main term, the negative residue is

$$
-\left[s^{-1}\right] \alpha p(q+p \alpha)^{-1} \Gamma(1) \frac{1}{s \log q}=\frac{\alpha p}{L(q+p \alpha)} .
$$

The fluctuations come from the negative residues of the poles at $s=\chi_{k}, k \neq 0$. Let $\varepsilon:=s-\chi_{k}$, then around $\varepsilon=0$ we get $\frac{1}{1-q^{-s}} \sim \frac{1}{\varepsilon \log q}$, and so these poles contribute:

$$
-\sum_{k \neq 0}\left[\varepsilon^{-1}\right] \alpha p(q+p \alpha)^{-\left(\chi_{k}+1\right)} \Gamma\left(\chi_{k}+1\right) \frac{1}{\varepsilon \log q} x^{-\chi_{k}}=\frac{\alpha p}{L(q+p \alpha)} \sum_{k \neq 0} \Gamma\left(1-\chi_{k}\right) e^{2 k \pi i \log _{Q}(x(q+p \alpha))}
$$

This gives a total probability in the (strict, weak) case asymptotic to

$$
\frac{\alpha(Q-1)}{L(1+\alpha(Q-1))}(1+\psi(n(q+p \alpha))),
$$

as $n \rightarrow \infty$ where $\psi(x)=\sum_{k \neq 0} \Gamma\left(1-\chi_{k}\right) e^{2 k \pi i \log _{Q} x}$.

(For $\alpha=1$, the dominant term gives $\frac{p}{L}$, which is the same as the probability of having one winner among $n$ players in a game where each player tosses a coin until a head appears, and the winner is the player who takes the longest to toss a head, see [3].)

\subsection{Case (weak, weak), for $\boldsymbol{d}=\boldsymbol{\alpha} n$}

Here, the maximum can recur anywhere, having first appeared at least once in the first $d$ letters. From [1], and by (2), we can approximate as in the (w,s) case to get:

$$
f_{(w, w)}(n) \sim \sum_{k \geq 1}\left[\left(e^{-n q^{k}}-1\right)-\left(e^{-n q^{k-1}(q+\alpha p)}-1\right)\right] .
$$

We define an exact function in terms of $x$ to be:

$$
f_{w w}(x):=\sum_{k \geq 1}\left[\left(e^{-x q^{k}}-1\right)-\left(e^{-x q^{k-1}(q+\alpha p)}-1\right)\right] .
$$


The transform of this function is

$$
\begin{aligned}
f_{w w}^{*}(s) & =\sum_{k \geq 1}\left[q^{-s k} \Gamma(s)-\left(q^{k-1}\right)^{-s}(q+\alpha p)^{-s} \Gamma(s)\right] \\
& =\Gamma(s)\left[1-q^{s}(q+\alpha p)^{-s}\right] \frac{1}{q^{s}-1},
\end{aligned}
$$

which exists in the strip $\langle-1,0\rangle$. We can thus rewrite $f_{w w}(x)$ as a contour integral

$$
f_{w w}(x)=\frac{1}{2 \pi i} \int_{\left(-\frac{1}{2}\right)} \Gamma(s)\left[1-q^{s}(q+\alpha p)^{-s}\right] \frac{1}{q^{s}-1} x^{-s} d s .
$$

The relevant simple poles occur at $s=0$ and $s=\chi_{k}, k \neq 0$. The negative residue at $s=0$ is

$$
-\left[s^{-1}\right] \frac{1}{s} s \log \left(\frac{q+\alpha p}{q}\right) \frac{1}{s \log q}=\frac{1}{L} \log \left(\frac{q+\alpha p}{q}\right),
$$

which, as in the (weak, strict) case, is one for $\alpha=1$. For the poles at $s=\chi_{k}$, let $\varepsilon:=s-\chi_{k}$. Then expanding around $\varepsilon=0$ gives $\frac{1}{q^{s}-1} \sim \frac{1}{\varepsilon \log q}$ and so the negative residues are

$$
-\sum_{k \neq 0}\left[\varepsilon^{-1}\right] \Gamma\left(\chi_{k}\right)\left[1-q^{\chi_{k}}(q+\alpha p)^{-\chi_{k}}\right] \frac{1}{\varepsilon \log q} x^{-\chi_{k}}=\frac{1}{L} \sum_{k \neq 0} \Gamma\left(-\chi_{k}\right)\left[e^{2 k \pi i \log _{Q} x}-e^{2 k \pi i \log _{Q}\left(x\left(\frac{q+\alpha p}{q}\right)\right)}\right] .
$$

By summing (14) and (15) and replacing $x$ by $n$ the asymptotic result for the (weak, weak) case in Theorem 1 is found.

\section{Suppose $1 \leq d=o(n)$}

Initially, in this section $d$ was considered to be dependent on $n$ according to the relationship $d=\alpha n^{\gamma}$, where $0<\gamma<1$, and $0<\alpha \leq 1$. Mellin transforms were used to obtain the same results as found in the $d$ fixed case (see [1]). However, it was then found (as suggested by a referee) that in fact any $d$ such that $1 \leq d=o(n)$ will produce the same results. The explanation is given below.

We show that the results are the same as when $d$ is fixed by referring back to the step in the calculations for $d$ fixed (see [1]) where the $d=\alpha n$ calculations failed. The important stage is when the main term of the probability is given by the expression

$$
\sum_{i=0}^{d-1} \sum_{l=0}^{d-1-i}\left(\begin{array}{c}
d-1-i \\
l
\end{array}\right)(-1)^{l} \frac{Q^{-l}\left(1-Q^{-1}\right)}{L} \frac{1}{(l+1+N)\left(\begin{array}{c}
N+l \\
l
\end{array}\right)} .
$$

This is the (weak, strict) case, but the others are similar. Since $N$ grows like $n$, we use $n$ instead of $N$ for simplicity. For $d$ fixed, it can be seen that the $l=0$ term dominates, since each term in the sum on $l$ is of order $O\left(\frac{1}{n^{l+1}}\right)$. For $d$ proportional to $n$, each term in the inner sum is of order $O\left(\frac{1}{n}\right)$, so none clearly dominates, and Mellin transforms are required to find the result (see Section 3). But what if $d=\alpha n^{\gamma}$ for $0<\gamma<1$, or even $d=\frac{n}{\log n}$ ?

Suppose we let $f(n)=o(n)$ for some $f(n)$ such that $f(n) \rightarrow \infty$ as $n \rightarrow \infty$. Then we can write $d=\frac{n}{f(n)}(=o(n))$. In general, a typical term in the sum on $l$ is of order $O\left(\frac{1}{n f^{l}(n)}\right)$. For $l=0$, we again have an order of $O\left(\frac{1}{n}\right)$. In fact, this term can be expressed as $\frac{c}{n}$ where $c$ is a constant. This will dominate all other terms, since even the infinite sum on $l$ (a geometric series) is:

$$
\frac{1}{n} \sum_{l=1}^{\infty}(f(n))^{-l}=\frac{1}{n(f(n)-1)}=o\left(\frac{1}{n}\right) .
$$

The results in Theorem 2 follow from the above.

\section{Conclusion}

Table 1 is a summary of results from this paper. (The $d$ fixed results are from [1]). This table shows only the dominant term for the results, expressed in terms of $Q$. 


\begin{tabular}{|c|c|c|c|c|}
\hline Case & $(\mathrm{s}, \mathrm{s})$ & $(\mathrm{w}, \mathrm{s})$ & $(\mathrm{s}, \mathrm{w})$ & $(\mathrm{w}, \mathrm{w})$ \\
\hline $1 \leq d=o(n)$ & $\frac{\left(1-Q^{-1}\right) d}{L n}$ & $\frac{\left(1-Q^{-1}\right) d}{L n}$ & $\frac{(Q-1) d}{L n}$ & $\frac{(Q-1) d}{L n}$ \\
\hline$d=\alpha n$ & $\frac{\left(1-Q^{-1}\right) \alpha}{L}$ & $\frac{\log \left(1-\alpha\left(1-Q^{-1}\right)\right)}{-L}$ & $\frac{\alpha(Q-1)}{L(1+\alpha(Q-1))}$ & $\frac{\log (1+\alpha(Q-1))}{L}$ \\
\hline
\end{tabular}

Tab. 1: Table of results for the two catagories

If we consider $\alpha$ small (i.e., close to zero) in the second category, we should get similar solutions to catagory one (in which $d$ is always small relative to $n$ for $n$ large). We thus determine what these dominant terms look like asymptotically as $\alpha \rightarrow 0$. We use the approximations $\log (1+x) \sim x$ and $\frac{1}{1-x} \sim 1$ as $x \rightarrow 0$ ([4]). Suppose $d=\alpha n$, then for the (weak, strict) case, we have

$$
\frac{\log \left(1-\alpha\left(1-Q^{-1}\right)\right)}{\log Q^{-1}} \sim \frac{-\alpha\left(1-Q^{-1}\right)}{\log Q^{-1}}=\frac{\alpha\left(1-Q^{-1}\right)}{L} .
$$

For the (strict, weak) case, we find that

$$
\frac{\alpha(Q-1)}{L(1+\alpha(Q-1))} \sim \frac{\alpha(Q-1)}{L},
$$

and for the (weak, weak) case,

$$
\frac{\log (1+\alpha(Q-1))}{L} \sim \frac{\alpha(Q-1)}{L} .
$$

By replacing each $\alpha$ by $\frac{d}{n}$, it can be seen that each of these corresponds to the $d$ fixed case in Table 1 above.

\section{Acknowledgements}

I hereby acknowledge the continued support, help and availability of my supervisors Prof. H. Prodinger and Prof. A. Knopfmacher. I would also like to acknowledge the referee for many helpful comments.

\section{References}

[1] M. Archibald. Restrictions on the position of the maximum/minimum in a geometrically distributed sample. In Mathematics and Computer Science III: Algorithms, Trees, Combinatorics and Probabilities, September 2004.

[2] P. Flajolet and R. Sedgewick. Analytic combinatorics, symbolic combinatorics (chapters I-IX). http://pauillac.inria.fr/algo/flajolet/Publications/books.html, November 2004.

[3] P. Kirschenhofer and H. Prodinger. The number of winners in a discrete geometrically distributed sample. Annals in Applied Probability, 6:687-694, 1996.

[4] R. Sedgewick and P. Flajolet. An Introduction to the Analysis of Algorithms. Addison-Wesley, 1996.

[5] W. Szpankowski. Average Case Analysis of Algorithms on Sequences. John Wiley and Sons, New York, 2001. 